(C) 2021, The Authors. Published by Elsevier Inc. and Fass Inc. on behalf of the American Dairy Science Association ${ }^{\circledR}$. This is an open access article under the CC BY-NC-ND license (http://creativecommons.org/licenses/by-nc-nd/4.0/).

\title{
Indirect genomic predictions for milk yield in crossbred Holstein-Jersey dairy cattle
}

\author{
Y. Steyn, ${ }^{1 *} \odot$ D. Gonzalez-Pena, ${ }^{2} \odot$ Y. L. Bernal Rubio, ${ }^{2} \odot$ N. Vukasinovic, ${ }^{2} \odot$ S. K. DeNise, ${ }^{2}$ \\ D. A. L. Lourenco, ${ }^{1}{ }^{\infty}$ and I. Misztal ${ }^{1}\left({ }^{\circ}\right.$ \\ ${ }^{1}$ Department of Animal and Dairy Science, University of Georgia, 425 River Road, Athens 30602 \\ ${ }^{2}$ Zoetis, 333 Portage Street, Kalamazoo, MI 49007
}

\begin{abstract}
The objective of this study was to predict genomic breeding values for milk yield of crossbred dairy cattle under different scenarios using single-step genomic BLUP (ssGBLUP). The data set included 13,880,217 milk yield measurements on $6,830,415$ cows. Genotypes of 89,558 Holstein, 40,769 Jersey, and 22,373 Holstein-Jersey crossbred animals were used, of which all Holstein, 9,313 Jersey, and 1,667 crossbred animals had phenotypic records. Genotypes were imputed to 45K SNP markers. The SNP effects were estimated from single-breed evaluations for Jersey (JE), Holstein (HO) and crossbreds (CROSS), and multibreed evaluations including all Jersey and Holstein (JE_HO) or approximately equal proportions of Jersey, Holstein, and crossbred animals (MIX). Indirect predictions (IP) of the validation animals (358 crossbred animals with phenotypes excluded from evaluations) were calculated using the resulting SNP effects. Additionally, breed proportions (BP) of crossbred animals were applied as a weight when IP were estimated based on each pure breed. The predictive ability of IP was calculated as the Pearson correlation between IP and phenotypes of the validation animals adjusted for fixed effects in the model. Regression of adjusted phenotypes on IP was used to assess the inflation of IP. The predictive ability of IP for CROSS, JE, HO, JE_HO, and MIX scenario was $0.50,0.50,0.47,0.50$, and 0.46 , respectively. Using $\mathrm{BP}$ was the least successful, with a predictive ability of 0.32. The inflation of the IP for crossbred animals using CROSS, JE, HO, JE_HO, MIX, and BP scenarios were $1.17,0.65,0.55,0.78,1.00$, and 0.85 , respectively. The IP of crossbred animals can be predicted using singlestep GBLUP under a scenario that includes purebred genotypes.
\end{abstract}

Received August 11, 2020.

Accepted January 5, 2021.

*Corresponding author: yvette.steyn@uga.edu
Key words: single-step GBLUP, breed proportions, direct genomic value, independent chromosome segments

\section{INTRODUCTION}

Genomic breeding values are typically estimated within pure breeds, especially in dairy cattle. Interest in combined purebred and crossbred evaluation is limited to countries with a large number of crossbred animals that are potentially used for breeding, for example, in New Zealand (Harris and Johnson, 2010). As of May 2019 , the total number of genotyped dairy cattle in the United States exceeded 3 million; only about $2 \%$ of those genotypes were of crossbred cattle (VanRaden et al., 2020). By September 2020, the number of genotyped US dairy animals increased to over 4.5 million, of which $86 \%$ are Holstein and $12 \%$ are Jersey (CDCB, 2020a). Although the proportion of crossbred animals is small, it amounts to a substantial financial cost that requires a return in investment. Crossbreds are becoming increasingly popular. An analysis of trends in the breed composition of US Dairy Herd Improvement herds showed that the percentage of dairy cattle reported as crossbred increased from 0.1 to $5.3 \%$ from 1990 to 2018 (Guinan et al., 2019). In April 2019, the Council on Dairy Cattle Breeding (CDCB) extended genomic evaluation services to provide estimates for crossbred animals (Wiggans et al., 2019, CDCB, 2020b).

Joint modelling of purebreds and crossbreds may require adjustments to account for nonadditive effects and heterogeneous variance of the breeds (Wei and van der Werf, 1994; Christensen et al., 2014). For purebred parents and $\mathrm{F}_{1}$ crossbred animals, an optimum strategy may be based on separating the genomic information for $F_{1}$ due to each parent. However, simpler methods based on combining all genotypes in a single relationship matrix may work as well (Lourenco et al., 2016). Another possibility is providing evaluations based on purebreds and estimated breed proportions (VanRaden et al., 2020). 
Genomic evaluation works primarily by estimating the value of chromosome segments (Daetwyler et al., 2010; Habier et al., 2013; Pocrnic et al., 2016a, 2019a). Such segments are different for each pure breed and probably only partially overlap with those of purebreds for crossbreds. In such cases, it would be useful to include the crossbred data for crossbred prediction.

Single-step genomic BLUP (ssGBLUP) is widely used for different species in numerous countries. This method includes both genotyped and nongenotyped animals independently of the phenotyping status (Legarra et al., 2009). Therefore, it allows the joint evaluation of purebred and crossbred animals, as well as separate evaluations. Additionally, there is an interest to apply genomic selection in commercial crossbred animals that may not be part of the official evaluation (i.e., not registered). These animals could have indirect genomic predictions (IP) computed based on SNP effects backsolved from the official evaluation. The objective of this study was to evaluate the predictive ability and inflation of indirect genomic predictions for crossbred animals using SNP effects estimated with ssGBLUP methods. Genotypes of Holsteins, Jerseys, and Holstein-Jersey crossbreds were used in ssGBLUP for the estimation of SNP effects. Additional comparisons involved a method that incorporates breed proportions based on genomic information.

\section{MATERIALS AND METHODS}

The trait of interest was milk yield, and 13,880,217 records were available on 6,830,415 animals, consisting of Holstein, Jersey, and animals classified as HolsteinJersey crossbreds. Phenotypic and pedigree data were directly obtained from producers in the United States through on-farm software using proprietary scripts. Genotypes were obtained from the Zoetis Genotyping Lab (Kalamazoo, MI), and a variety of low-density chips with several SNP (ranging from about 3,000 to over 35,000 , and medium-density chips with 50 to $80 \mathrm{~K}$ markers) were used (Illumina Inc., 2011a,b). Animals genotyped on chips with less than 40K SNP were imputed using the program FImpute (Sargolzaei et al., 2011) to 45,245 markers. Details on the data sources is provided in Vukasinovic et al. (2017). The crossbred animals were imputed using a reference population of 795 Holstein-Jersey crossbred animals. The numbers of animals genotyped (and phenotyped) were 375,487 $(89,558)$ Holstein, 40,769 $(9,313)$ Jersey, and 22,373 $(1,667)$ crossbred animals. Among the parents of crossbred animals, 1,331 Holstein sires and 202 Holstein dams, 147 Jersey sires, and 122 Jersey dams were genotyped. In total, $9 \%$ of the purebred parents of crossbred animals were genotyped. Breed proportions (BP) were

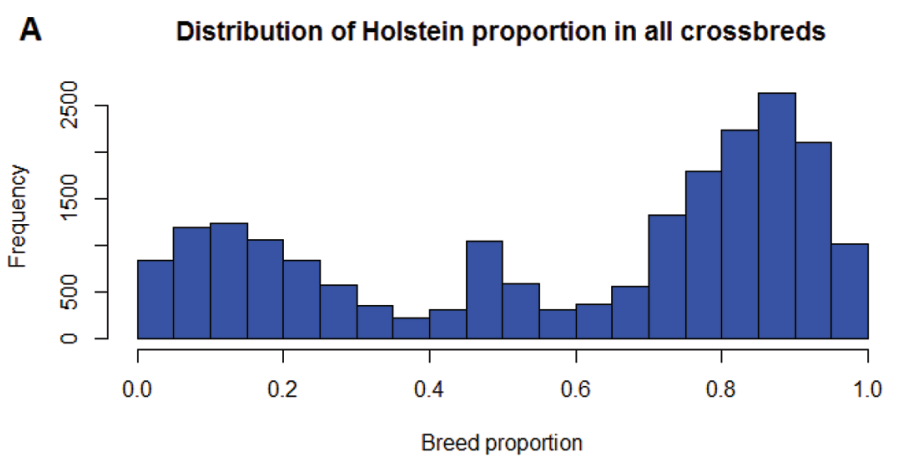

B Distribution of Holstein proportion in validation crossbreds

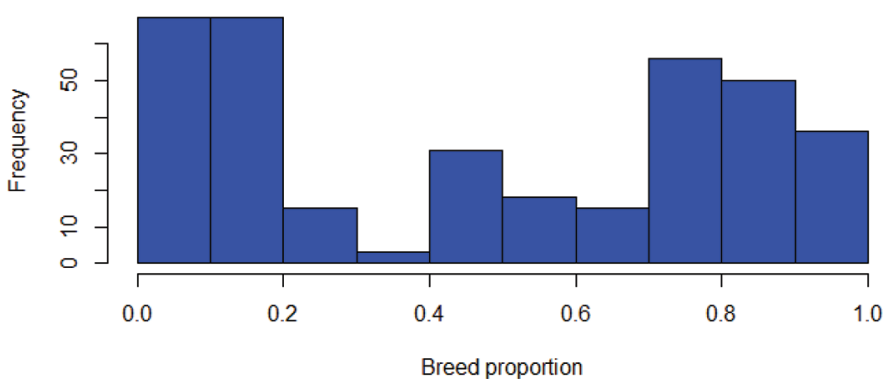

Figure 1. Distribution of the proportion of the crossbred genotypes that were assigned as Holstein. Panel A applies to all crossbred animals, whereas panel B applies only to validation crossbred animals.

obtained for crossbreds based on genotypes using an Admixture (Alexander et al., 2009) analysis supervised with 2 clusters. The available crossbreds were of varying breed proportions (1-99\% Holstein or Jersey). Figure $1 \mathrm{~A}$ shows the distribution for the Holstein proportion in all crossbred animals. The average Holstein BP for all genotyped crossbred animals was $59 \%$, and Jersey BP was $41 \%$. Figure 2 shows the first 2 principal components for all crossbred animals. All genotyped Jersey and crossbred animals were used, and 89,558 Holstein animals with phenotypes and genotypes were selected to lower computational requirements.

Validation animals for milk yield consisted of animals that had measurements for the first lactation only and were born within 2015 to 2017, such that the validation populations were $20 \%$ of all the genotyped animals. There were 3 different validation populations: Holstein (15,695 animals), Jersey (2,186 animals), and crossbreds (358 animals). Among the parents of the 358 validation crossbred animals, 61 Holstein sires and 10 Holstein dams, and 26 Jersey sires and 19 Jersey dams were genotyped. This amounted to $32 \%$ of the known purebred parents of crossbred validation animals. Breed proportions of validation animals ranged from 1 to $99 \%$ of either Holstein or Jersey. Figure 1B shows the distribution of the proportion of Holstein in validation cross- 


\section{PC plots of crossbred animals}

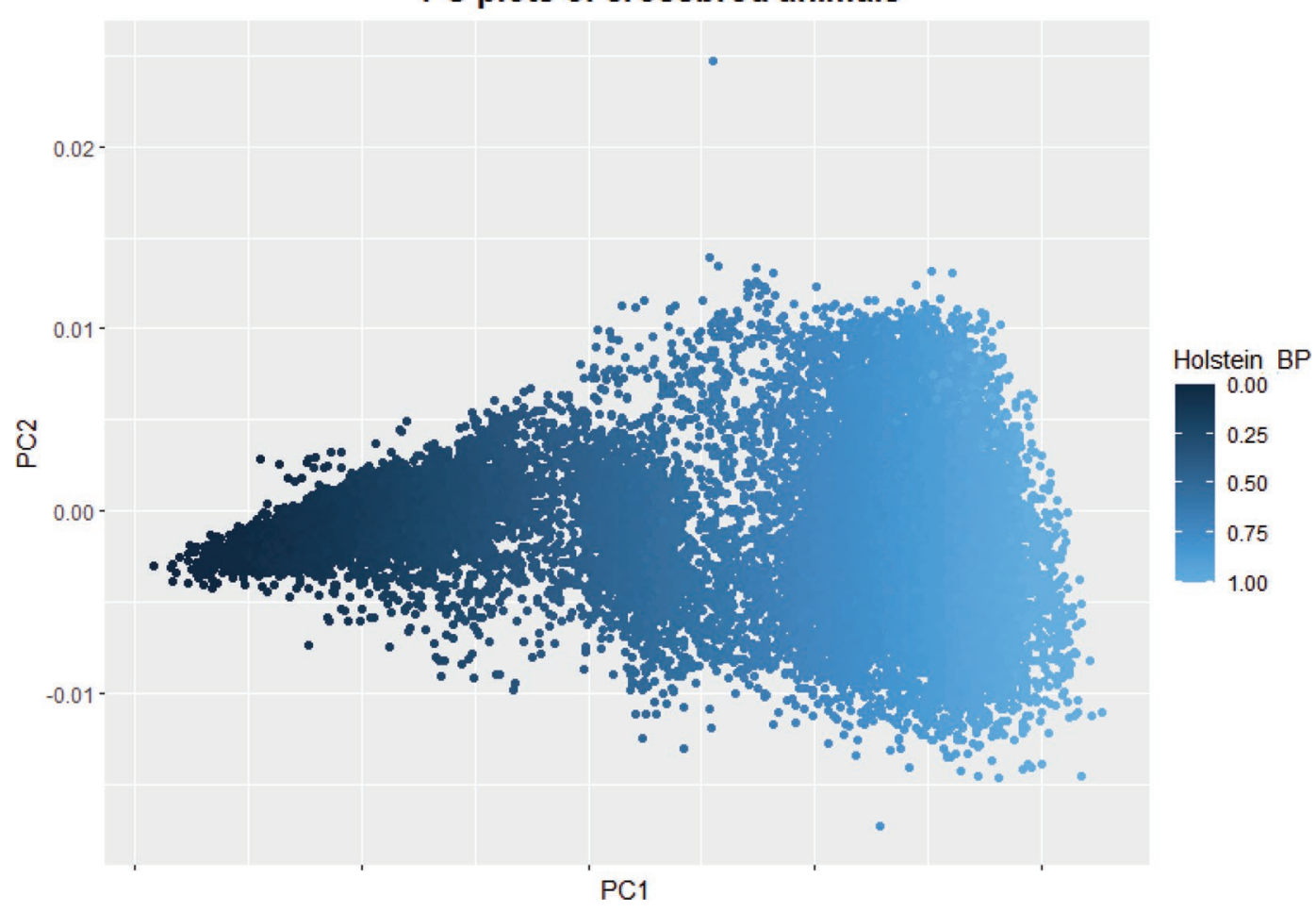

Figure 2. Principal component (PC) plot for all crossbred animals. Color intensity indicates the Holstein breed proportion (BP) of each crossbred animal. Animals with a Holstein BP $<0.50$ have higher Jersey BP.

bred animals. The average Holstein BP for crossbred validation animals was $48 \%$, and Jersey BP was $52 \%$. Phenotypes of the validation populations were removed to estimate SNP effects based on that breed/group. All evaluations included the same pedigree and data file of all available animals, but the selection of genotypes differed for each scenario.

The repeatability animal model for milk yield was

$$
\mathbf{y}=\mathbf{X b}+\mathbf{Z}_{1} \mathbf{u}+\mathbf{Z}_{2} \mathbf{p e}+\mathbf{Z}_{3} \mathbf{h s}+\mathbf{e}
$$

where $\mathbf{y}$ is the vector of milk yield, $\mathbf{X}$ is the incidence matrix assigning the measurement to fixed effects that included age group, management group, and pedigreebased inbreeding and heterosis, obtained using the $\mathrm{R}$ package optiSel (Wellmann, 2019, 2020); b is a vector of solutions for fixed effects, $\mathbf{Z}_{1}$ is the incidence matrix assigning the measurement to the random animal effect; $\mathbf{u}$ is a vector of solutions for animal, $\mathbf{Z}_{2}$ is the incidence matrix for random permanent environment effect; pe is a vector of solutions for permanent environment effect; $\mathbf{Z}_{3}$ is an incidence matrix for the random effect of sire nested within herd; hs is a vector of solutions for herd $\times$ sire interaction, and $\mathbf{e}$ is the residual. It was assumed that $\mathbf{u} \sim N\left(0, \mathbf{H} \sigma_{u}^{2}\right)$, where $\sigma_{u}^{2}=$ addi- tive genetic variance, and $\mathbf{H}$ is the relationship matrix combining genotyped and nongenotyped animals in ssGBLUP (Legarra et al., 2009); pe $\sim N\left(0, \mathbf{I} \sigma_{p e}^{2}\right)$, where $\sigma_{p e}^{2}=$ permanent environment variance; $\mathbf{h s} \sim N\left(0, \mathbf{I} \sigma_{h s}^{2}\right)$, where $\sigma_{h s}^{2}=$ herd $\times$ sire variance; $\mathbf{e} \sim N\left(0, \mathbf{I} \sigma_{e}^{2}\right)$; and $\mathbf{I}$ is the identity matrix. A heritability of 0.30 was assumed for milk yield (Wiggans, 1997).

The inverse of $\mathbf{H}$, which is required for the ssGBLUP evaluations, was constructed as in Aguilar et al. (2010):

$$
\mathbf{H}^{-1}=\mathbf{A}^{-1}+\left[\begin{array}{cc}
0 & 0 \\
0 & \mathbf{G}^{-1}-\mathbf{A}_{22}^{-1}
\end{array}\right] .
$$

In this study, $\mathbf{G}$ was obtained using the formula $\mathbf{G}=\frac{\mathbf{M M}^{\prime}}{2 \sum p_{i}\left(1-p_{i}\right)}$, where $\mathbf{M}$ is a matrix of SNP content centered by twice the current allele frequencies, and $p_{i}$ is the allele frequency for SNP $i$ (VanRaden, 2008). The pedigree-based relationship matrix between genotyped animals is referred to as $\mathbf{A}_{22}$. To reduce bias due to the different genetic level of genotyped and nongenotyped animals, $\mathbf{G}$ was tuned to be compatible with 
$\mathbf{A}_{22}$, using the method described by Chen et al. (2011). To avoid singularity problems, $5 \%$ of $\mathbf{A}_{22}$ was combined with $95 \%$ of $\mathbf{G}$.

The $\mathbf{G}$ was constructed differently for each scenario to account for the difference in allele frequencies. Three evaluations were within-breed: Jersey (JER), Holstein (HOL), and crossbreds (CROSS). The respective G matrices were constructed using within-breed allele frequencies. Two evaluations were multibreed and used the relevant genotypes to construct different $\mathbf{G}$ matrices: (1) all Jersey and Holstein genotypes used as reference (JER_HOL) with G constructed using allele frequencies of all Jersey and Holstein combined; and (2) equal proportions $(\sim 20 \mathrm{~K}$ each) of Jersey, Holstein and crossbreds (MIX) with $\mathbf{G}$ constructed using allele frequencies of only these animals. Because the number of genotyped animals was large in all scenarios, except CROSS, the inverse of $\mathbf{G}$ was computed using the APY algorithm, with core animals selected randomly (Fragomeni et al., 2015; Misztal, 2016). The number of core animals corresponded approximately to the number of eigenvalues explaining $99 \%$ variation in $\mathbf{G}$. The number of eigenvalues explaining 90, 95, 98, and 99\% are presented in Table 1. The direct inverse of $\mathbf{G}$ was only used for the CROSS evaluation.

Single-step genomic BLUP implemented in the BLUPF90 software suite (Misztal et al., 2014) was used for analyses of these 5 scenarios. After removing phenotypes of all validation animals, genomic breeding values (GEBV) for all animals were estimated in each scenario. The BLUP90IOD2 v3.102 software (Misztal et al., 2014) was used to compute these GEBV. The combination of breeds and groups together assumes that SNP effects and variances are the same across all populations. The SNP effects were estimated for each scenario separately based on these GEBV, using the POSTGSF90 v1.63 software package with the formula (VanRaden, 2008; Wang et al., 2012)

$$
\hat{\mathbf{a}}=\lambda \mathbf{D} \mathbf{M}^{\prime} \mathbf{G}^{-1}(\mathrm{GEBV}),
$$

where $\hat{\mathbf{a}}$ is a vector of estimated SNP effects, $\lambda$ is the ratio of SNP to additive genetic variance, $\mathbf{D}$ is a diagonal matrix of weights for SNP (in this case, an identity matrix), and $\mathbf{M}$ was as defined before. The $\mathbf{G}^{-1}$ for JER, HOL, JER_HOL, and MIX were obtained using APY. The IP from ssGBLUP have been shown to be stable when using different core animals as long as the size of the core is at least equal to the number of eigenvalues required to explain 98 to $99 \%$ of the genomic variation (Garcia et al., 2020), which was the case in this study.

Based on SNP effects, IP for validation animals were calculated as the sum of SNP effects weighted by the genotype content using the PREDF90 v1.04 software (Misztal et al., 2014). Across-breed predictions were obtained using SNP effects estimated in one scenario to predict the IP of validation animals not included in that evaluation. The IP obtained using different methods were compared with the GEBV obtained with full data in the CROSS ( $\left.\mathrm{GEBV}_{\text {Full_CROSS }}\right)$ and MIX $\left(\mathrm{GEBV}_{\text {Full_MIX }}\right)$ scenarios, as well as GEBV obtained using CROSS without data of the validation populations $\left(\mathrm{GEBV}_{\text {Partial }}\right)$.

Indirect predictions for crossbred animals were also estimated using BP and IP obtained using SNP effects of both the component breeds with the following formula:

$$
\mathrm{IP}_{\mathrm{BP}}=\mathrm{BP}_{\mathrm{H}}\left(\mathrm{IP}_{\mathrm{H}}\right)+\mathrm{BP}_{\mathrm{J}}\left(\mathrm{IP}_{\mathrm{J}}\right),
$$

where $\mathrm{IP}_{\mathrm{BP}}$ is the IP of crossbred animals using breed proportions, $\mathrm{BP}_{\mathrm{H}}$ is the proportion of Holstein in the crossbred animal, $\mathrm{BP}_{\mathrm{J}}$ is the proportions of Jersey in the crossbred animal, $\mathrm{IP}_{\mathrm{H}}$ is the IP of the crossbred animal estimated using Holstein SNP effects, and $\mathrm{IP}_{\mathrm{J}}$ is the IP of the same crossbred animal estimated using Jersey SNP effects. This weighting of the IP with the BP follows the same concept as VanRaden et al. (2020) and Strandén and Mäntysaari (2013). A requirement for this method is for IP to be on an all-breed scale, which

Table 1. Eigenvalues explaining $90,95,98$, and $99 \%$ of variation in the genomic relationship matrix $(\mathbf{G})$ when

\begin{tabular}{|c|c|c|c|c|c|}
\hline \multirow[b]{2}{*}{ Breed } & \multirow[b]{2}{*}{ Animals } & \multicolumn{4}{|c|}{ Variation } \\
\hline & & $90 \%$ & $95 \%$ & $98 \%$ & $99 \%$ \\
\hline Holstein & 89,558 & 4,637 & 8,101 & 14,068 & 19,046 \\
\hline Jersey & 40,769 & 3,325 & 5,775 & 9,841 & 13,216 \\
\hline Cross & 22,373 & 4,939 & 6,698 & 8,676 & 9,887 \\
\hline Jersey and Holstein & 130,327 & 5,661 & 9,666 & 16,250 & 21,564 \\
\hline MIX $^{1}$ & 61,275 & 7,396 & 11,857 & 18,017 & 22,483 \\
\hline
\end{tabular}
all genotyped animals are considered

${ }^{1}$ The mixed population contains approximately equal numbers of Holstein, Jersey, and crossbred animals. 
Table 2. Predictive ability (Pearson correlation between indirect predictions and adjusted phenotype) when using marker effects based on a breed, or group, to predict the indirect genomic value of itself and that of others $^{1}$

\begin{tabular}{|c|c|c|c|c|}
\hline \multirow[b]{2}{*}{ Breed used } & \multicolumn{3}{|c|}{ Predictive ability } & \multirow{2}{*}{$\frac{\text { Inflation }}{\text { Cross }}$} \\
\hline & Jersey & Holstein & Cross & \\
\hline Jersey & $0.48^{2}$ & 0.13 & 0.50 & 0.65 \\
\hline Holstein & 0.09 & $0.45^{2}$ & 0.47 & 0.55 \\
\hline Cross & 0.24 & 0.26 & $0.50^{2}$ & 1.17 \\
\hline Jersey and Holstein & $0.45^{2}$ & $0.44^{2}$ & 0.50 & 0.78 \\
\hline Mix & $0.46^{2}$ & $0.40^{2}$ & $0.46^{2}$ & 1.00 \\
\hline Proportions & - & - & $0.32^{2}$ & 0.85 \\
\hline
\end{tabular}

was achieved by including phenotypes of all breeds and a full pedigree in every evaluation.

The predictive ability was determined using a Pearson correlation between the phenotype adjusted for fixed and other random effects, and the IP of the relevant validation population. The adjusted phenotype was obtained using the PREDICTf90 v1.3 software package, with the same model as described before, and data of all animals, regardless of breed. No genotypes were included for this purpose. Inflation was measured as the regression coefficient when regressing adjusted phenotype on IP. A coefficient of 1 indicates no inflation, $>1$ indicates an under-estimation (deflation), and $<1$ an over-estimation (inflation).

\section{RESULTS}

The numbers of eigenvalues required to explain 90, 95, 98 , and $99 \%$ of the variation in the $\mathbf{G}$ matrix for the different scenarios are presented in Table 1. To apply APY, it is recommended to have a core size equal to the number of eigenvalues that explains between 98 and $99 \%$ of the variation of $\mathbf{G}$ (Pocrnic et al., 2016b). Because available resources were able to handle larger core sizes, the core was selected to explain at least $99 \%$.

Results of the predictive abilities of indirect predictions are presented in Table 2 . The predictive ability within breed was $0.48,0.45$, and 0.50 for JER, HOL, and CROSS, respectively. The JER could not predict HOL well (0.13), and HOL had even lower ability to predict JER (0.09), which was expected because across-breed predictions have been shown to have low accuracies in dairy cows (Pryce et al., 2011; Olson et al., 2012). Both pure breeds could predict CROSS approximately the same as when using only crossbred animals (0.50 with JER effects, 0.47 with HOL, and 0.50 with CROSS). The CROSS was better at predicting the pure breeds (0.24 for JER and 0.26 for HOL) than the pure breeds were able to predict each other (across-breed); however, the predictive ability was still low. The JER_HOL scenario could predict all 3 groups with relatively similar predictive ability as the single-breed analyses (0.45, 0.44 , and 0.50 for Jersey, Holstein, and crossbreds, respectively). Making use of breed proportions was the least successful of all scenarios, with predictive ability equal to 0.32 .

The resulting IP for crossbred validation animals in the different scenarios were compared with the GE$\mathrm{BV}_{\text {Partial }}, \mathrm{GEBV}_{\text {Full_CROSS }}$, and GEBV $\mathrm{Full \_ MIX}_{\text {. Table } 3}$ summarizes these correlations. The estimated GEBV were adjusted for the genetic base consisting of animals born in 2015. The means were -70.13 for IP based on CROSS, -929.48 for $\mathrm{GEBV}_{\text {Partial }},-1003.76$ for $\mathrm{GEBV}_{\text {Full_CROSS }}$, and -1083.12 for GEBV $V_{\text {Full_MIX. }}$ Figure 3 compares the distribution of adjusted phenotype to the IP of crossbred animals obtained from different approaches and GEBV $V_{\text {Partial. }}$. The means and standard deviations differed between scenarios, especially when crossbred animals were not part of the reference population. The distribution of the adjusted phenotypes was wide, whereas distributions were more centered around 0 for the $\mathrm{GEBV}_{\text {Partial }}$ and IP when SNP effects were based on CROSS, BP, and MIX, and their ranges were narrower compared with the IP based on JER, HOL, or JER_HOL. The IP for crossbred validation animals were generally $>0$ when based on JER and $<0$ when based on HOL.

The inflation of the IP of crossbred animals when using SNP effects based on CROSS, JER, HOL, JER HOL, and MIX was 1.17, 0.65, 0.55, 0.78, and 1.00 . Inflation when IP was based on pure breeds or with breed proportions was 0.85 .

\section{DISCUSSION}

The number of eigenvalues associated with the genomic relationship matrix is an indicator of the number of independent chromosome segments $\left(\mathbf{M}_{\mathbf{e}}\right.$; Pocrnic et 
al., 2016a). A smaller $\mathrm{M}_{\mathrm{e}}$ indicates longer chromosome segments and less genetic diversity within the population. The Jersey breed required considerably fewer eigenvalues compared with the Holstein. However, this may be an artifact of the number of genotypes available for Jersey. In the case of JER_HOL, more eigenvalues were required compared with either JER or HOL but less than the sum of eigenvalues obtained for those pure breeds separately. This deviation from the sum suggests that the Holstein and Jersey breeds share similarities (Pocrnic et al., 2019b), albeit not enough to do across-breed predictions. It is not unexpected for breeds, especially the ones with similar breeding goals (such as dairy breeds), to share similarities, such as the DGAT1 gene (Spelman et al., 2002; Thaller et al., 2003). In fact, genetic similarities can occur across species (Raymond et al., 2020). The number of eigenvalues required in the MIX scenario was considerably higher than that in the other scenarios. Because crossbred animals receive their genes from the component pure breeds, adding the crossbred genotypes is not expected to provide any additional genomic content information not already captured by purebred parents. However, crossbred animals in this study have a wide range of breed proportions instead of only $\mathrm{F}_{1}$. This may produce new haplotypes, recombination, and linkage disequilibrium not present in the pure breeds, thereby resulting in the higher number of eigenvalues needed to explain 98 to $99 \%$ of variation. More importantly, only a small proportion of known purebred parents were genotyped in our study. Thus, our crossbred population provided additional information from purebred animals that do not have genotypes. The study by Pocrnic et al. (2019b) on pigs found that adding $\mathrm{F}_{1}$ crossbred animals did not increase the number of eigenvalues beyond that of the purebred animals. However, the size of their data was larger than the $\mathrm{M}_{\mathrm{e}}$ in pigs.
The low ability of one pure breed to predict the other has been observed in previous work, both on real and simulated data (Pryce et al., 2011; Olson et al., 2012; Raymond et al., 2018; Steyn et al., 2019). Results of our study showed that when both purebreds were combined in the JER_HOL multibreed evaluation, the predictive ability of the purebred validation animals was slightly lower than single-breed evaluations (0.01 lower for Holstein and to 0.03 for Jersey), which corresponded to other studies that have not found much of a difference (Pryce et al., 2011; Olson et al., 2012). The change in predictive ability for Holstein was small enough to be negligible. In multibreed evaluations, it is important for component breeds to be present in the reference population (Toosi et al., 2010; Pryce et al., 2011; Olson et al., 2012; Steyn et al., 2019). The small, but slightly larger, decrease in predictive ability for Jersey could be because the number of Holstein genotypes far outweighs the number of Jersey genotypes in the JER_HOL reference population. When the number of genotypes per group is more balanced in the MIX scenario, the predictive ability of IP for Holstein decreased by 0.05 , but only decreased by 0.02 for Jersey IP compared with single-breed evaluations.

In the JER_HOL, the number of Holstein animals was considerably higher than the number of Jersey animals, yet the crossbred IP were predicted better than when all groups were represented equally (MIX). The ability to predict IP for Holstein or crossbreds noticeably decreased in the MIX scenario compared with singlebreed evaluations, whereas predictive ability of Jersey IP changed very little. The change in Holstein but not Jersey could be because the number of Holstein genotypes removed for the MIX scenario was proportionally larger for Holstein (from 90-20K) compared with the Jersey genotypes $(\sim 40-20 \mathrm{~K})$. More influential Holstein animals may have been removed, decreasing the aver-

Table 3. The correlations between indirect predictions of crossbred validation animals $\left(\mathrm{IP}_{\text {Cross }}\right)$ obtained using different SNP effects and genomic EBV (GEBV) of crossbred animals estimated with different data sets ${ }^{1}$

\begin{tabular}{|c|c|c|c|}
\hline $\mathrm{IP}_{\text {Cross }}$ & $\mathrm{GEBV}_{\text {Partial }}$ & GEBV $_{\text {Full_CROSS }}$ & GEBV $_{\text {Full_MIX }}$ \\
\hline \multicolumn{4}{|c|}{ Population used for SNP effects } \\
\hline Crossbreds & 0.97 & 0.86 & 0.73 \\
\hline Jersey & 0.75 & 0.72 & 0.65 \\
\hline Holstein & 0.68 & 0.66 & 0.62 \\
\hline Jersey and Holstein & 0.65 & 0.67 & 0.75 \\
\hline $\mathrm{Mix}^{2}$ & 0.76 & 0.72 & 0.84 \\
\hline \multicolumn{4}{|l|}{ Other method } \\
\hline Breed proportions & 0.29 & 0.37 & 0.56 \\
\hline
\end{tabular}



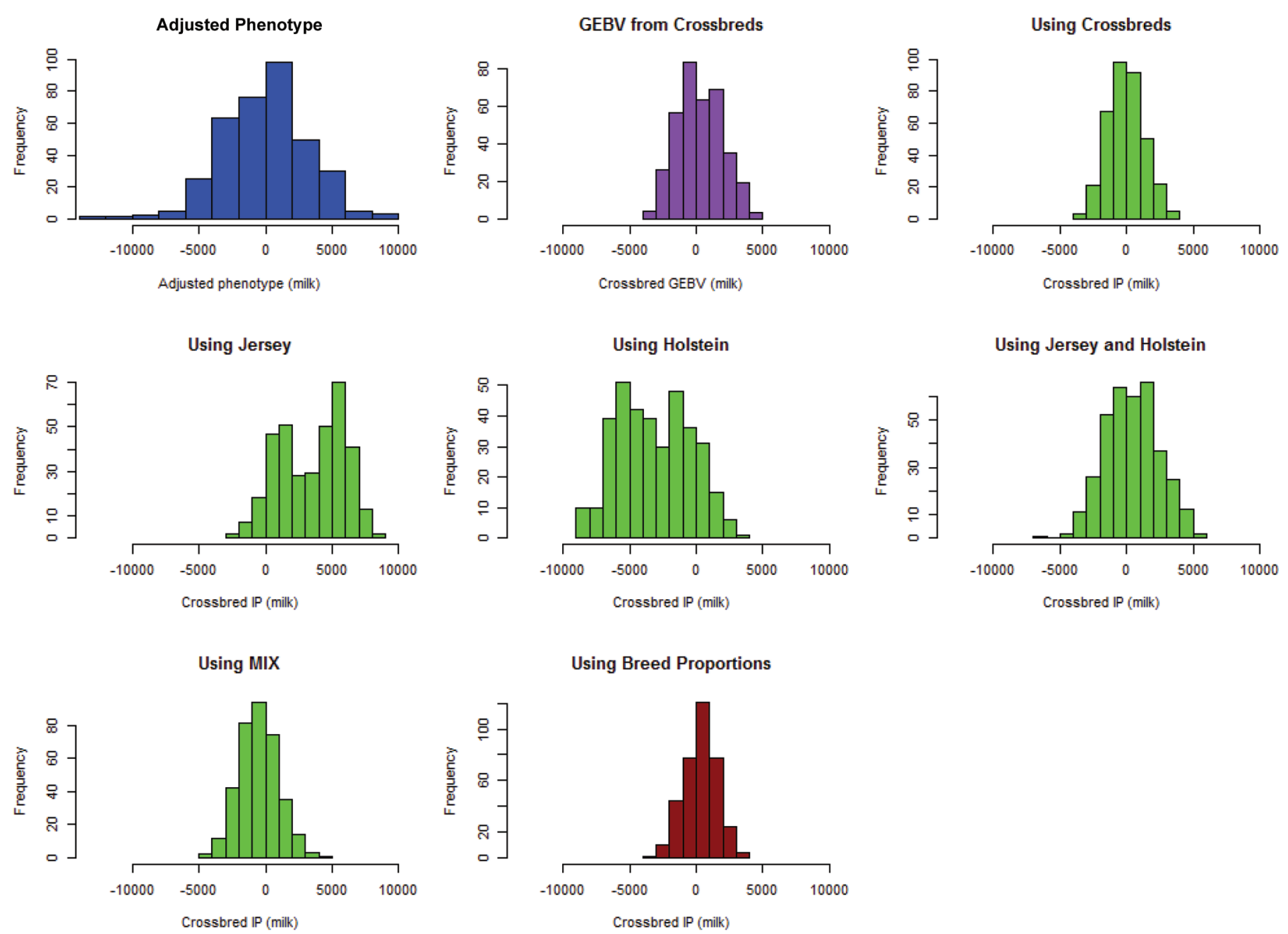

Figure 3. Distributions for the resulting estimations for crossbred validation animals. Distributions include adjusted phenotype, genomic breeding values (GEBV) obtained when genotypes of only crossbred animals were included and phenotypes of the validation populations were excluded, and indirect predictions (IP) obtained from SNP effects estimated when excluding phenotypes of validation animals and including genotypes of specific groups. The different reference groups to estimate SNP effects for the calculation of IP were composed of (1) only crossbred animals, (2) MIX (all the crossbred animals and equal proportions of each pure breed), (3) only Jersey genotypes, (4) only Holstein genotypes, or (5) both Jersey and Holstein genotypes. Another IP was obtained by using genomic breed proportions as weights to sum the IP obtained when using Jersey SNP effects, and when using Holstein SNP effects.

age relatedness between the MIX reference population and the Holstein validation populations. Additionally, the Jersey population may be genomically less diverse and more related to the MIX reference population.

In pigs, it was shown that the accuracy for crossbred animals was higher when using both parent breeds or equal number of animals from the parent breeds, compared with using either pure breed by itself or only crossbreds for most traits (Hidalgo et al., 2015). Pocrnic et al. (2019b) also found an increase in predictive ability for crossbred pigs when both pure lines were combined compared with only one pure breed. However, their study was on $\mathrm{F}_{1}$ crossbred animals and had more animals with both genotypes and phenotypes than our study. No increase in predictive ability of crossbred IP was observed in our study using JER_HOL compared with only using JER or CROSS, but there was a slight increase compared with using only HOL. The crossbreed structure of this study was complex with a range of breed proportions instead of $F_{1}$ crossbred animals. The purebred dairy breeds may also be genetically more diverse than the pig breeds, as reflected in the different effective population sizes in Pocrnic et al. (2016b). The MIX scenario included all the crossbred animals and equal proportions of each pure breed. This inclusion of purebred animals for crossbred evaluations was expected to yield higher predictive abilities, but this was not the case in our study. The random sampling of 
purebred animals may have excluded influential parents that contributed to previous predictive abilities.

In the study by VanRaden et al. (2020), the quality of prediction for crossbreds was evaluated by squared correlations of later milk yield deviation on earlier prediction by breed base representation. For all crossbred cows, those correlations for several traits were 0.01 to 0.05 higher for genomic predictions using breed proportions than for parent average, indicating limited improvement. Whereas the correlation was 0.05 higher for milk, it was much higher (up to 0.34) when separated by breed base representation. This shows the variation within crossbred evaluations, as the same approach to all can lead to different responses. Their study used much larger data for Holsteins and Jerseys, but crossbred animals were not used in the prediction process, only in validation.

In our study, accounting for breed proportions for the estimation of IP of crossbred animals led to a decrease in predictive ability compared with not making any adjustments at all (0.32 using proportions vs. 0.50 using CROSS). This does not correspond to VanRaden et al. (2020), in which using breed proportions was slightly more accurate than using the nearest pure breed. They also found that the accuracy was highest when the major breed proportion was from 75 to $90 \%$ (0.52), and lowest from 50 to $74 \%(0.35)$. The validation population in our study was, on average, $52 \%$ Jersey. The SNP effects in the study by VanRaden et al. (2020) were estimated from much larger data, whereas our study used approximately $2 \mathrm{~K}$ crossbred animals with both genotypes and phenotypes. The relatively high predictive ability of crossbreds based on the crossbred SNP effects in this study despite the small reference population could be due to the limited number of purebred parents.

Predictive abilities assume that the model was adequate to adjust for all effects other than the additive genetic effect. Breed effect was not specifically included in the model. Animals are generally compared withinbreed, and therefore breed adjustment will not compromise the ranking within the pure breed. Crossbred animals are most likely compared only with crossbreds to select replacement animals, but the range of breed proportions of animals adds a layer of complexity regarding breed effect. Accounting for nonadditive factors and accounting for breed origin of markers may increase the predictive ability for crossbred animals. However, Lopes et al. (2017) used a genomic matrix as described by Christensen et al. (2014) that takes breed effects into account for the estimation of GEBV before back-solving for SNP effects. They found that accounting for breed-specific effects did not change the accuracy of prediction compared with only using effects of crossbreds. Simulations showed that accounting for breed origin of alleles is only beneficial when lines are distantly related (Esfandyari et al., 2015a,b), which might be the case with Jersey and Holstein. Obtaining breed-specific effects in those studies required knowledge of the breed origin of alleles. Assigning breed of origin to alleles achieved accuracies $>90 \%$ in both simulated and real data (Sevillano et al., 2016; Vandenplas et al., 2016).

The IP of crossbred animals were inflated when using HOL (0.55), slightly less using JER (0.65), and even less when using JER_HOL (0.78). Using CROSS was the only scenario with a deflated IP of crossbred animals (1.17). Although using MIX had a lower predictive ability than using JER_HOL or CROSS, the IP were neither inflated nor deflated. Inflation when using breed proportions was 0.85 , which was better than using either pure breed or a combination of both, but not as successful as using MIX. This trade-off regarding predictive ability and inflation may influence the decision made by breed associations regarding the chosen reference population. Inflation in the study by VanRaden et al. (2020) was smaller when using breed proportions compared with using the nearest pure breed, which corresponds to this study.

Crossbred animals can be selected to be backcrossed with purebred animals with the objective to transform the current herd to a specific component pure breed (Holstein or Jersey in this study). In this case, the resulting progeny will be expected to perform within the genetic background of the pure breed. Therefore, SNP effects based on that pure breed could be useful (JER or HOL). The performance of crossbred is expected to be an intermediate of the parental breeds but often deviates from their average due to heterosis (Buckley et al., 2014). On average, Holstein milk production is considerably higher than Jersey milk production (CDCB, 2020c). This is reflected by the mean of the different IP, as presented in Figure 3. When IP are based on Jersey SNP effects for milk production, the IP for crossbreds are generally positive, whereas they are generally negative when using Holstein SNP effects. When IP were based on both Jersey and Holstein, the values were more intermediate.

The correlation between IP using CROSS and GE$B V_{\text {Partial }}$ was almost 1 , which was expected because these GEBV were used to estimate the SNP effects. These GEBV were not used to estimate SNP effects for all other scenarios. Therefore, correlations between the other IP and GEBV $V_{\text {Partial }}$ were considerably lower, but they were generally still high $(>0.65)$. The correlations between the IP from CROSS and GEBV Full_Cross and GEBV $V_{\text {Full_MIX }}$ were high (0.86 and 0.73) but lower than those with CROSS $_{\text {Partial. }}$. These correlations were 
expected to be lower because more information was available to improve the predictions. It is important for these correlations to still be high because high correlations show that the IP was a strong indicator of a future breeding value. All correlations with IP based on breed proportions were much lower, confirming that this approach was not appropriate for this data. However, the results are affected by sample size, and the number of crossbred animals in this data set was relatively small. There were also differences in means, as shown in Figure 3. To compare or rank animals using predictions obtained from different sources of SNP information, they can be transformed to the same scale (Legarra et al., 2018; Lourenco et al., 2018).

In this study, $<10 \%(24 \%)$ of the genotyped crossbred (purebred) animals had phenotypes. This is a challenge because the success of the estimation of SNP effects depends on the number of animals with both phenotypes and genotypes (Gonzalez-Recio et al., 2014); however, it is important to research methods to accurately predict breeding values of young animals based on information that is present before phenotypes become available.

\section{CONCLUSIONS}

Indirect predictions for crossbred animals can be computed via single-step GBLUP using various combinations of purebred and crossbred data. The best reference population considering predictive ability and inflation of prediction is the mix of purebred and crossbred animals. The IP themselves provide a useful tool to select crossbred animals.

\section{ACKNOWLEDGMENTS}

The authors acknowledge Zoetis Genetics for providing the data for this study. The research described herein was a part of the corresponding author's internship program sponsored through the research agreement between University of Georgia (Athens) and Zoetis. The authors have not stated any conflicts of interest.

\section{REFERENCES}

Aguilar, I., I. Misztal, D. Johnson, A. Legarra, S. Tsuruta, and T. Lawlor. 2010. Hot topic: a unified approach to utilize phenotypic, full pedigree, and genomic information for genetic evaluation of Holstein final score. J. Dairy Sci. 93:743-752. https://doi.org/10 $.3168 /$ jds.2009-2730.

Alexander, D. H., J. Novembre, and K. Lange. 2009. Fast model-based estimation of ancestry in unrelated individuals. Genome Res. 19:1655-1664. https://doi.org/10.1101/gr.094052.109.

Buckley, F., N. Lopez-Villalobos, and B. J. Heins. 2014. Crossbreeding: Implications for dairy cow fertility and survival. Animal 8(Suppl. 1):122-133. https://doi.org/10.1017/S1751731114000901.
CDCB. 2020a. Council on Dairy Cattle Breeding Activity Report Oct 19/Sep 2020. Accessed Dec. 2, 2020.https://www.uscdcb.com/wp -content/uploads/2020/10/2020-CDCB-Activity-Report_103020 _lowres.pdf.

CDCB. 2020b. Description of national genetic evaluation systems. Accessed Aug. 1, 2020. https://www.uscdcb.com/wp-content/ uploads/2020/04/USApro.pdf.

CDCB. 2020c. State and national standardized lactation averages by breed for cows calving in 2018. Accessed Oct. 8, 2020. https:// queries.uscdcb.com/publish/dhi/current/laall.shtml.

Chen, C.-Y.. I. Misztal, I. Aguilar, A. Legarra, and W. Muir. 2011. Effect of different genomic relationship matrices on accuracy and scale. J. Anim. Sci. 89:2673-2679. https://doi.org/10.2527/jas .2010-3555.

Christensen, O. F., P. Madsen, B. Nielsen, and G. Su. 2014. Genomic evaluation of both purebred and crossbred performances. Genet. Sel. Evol. 46:23. https://doi.org/10.1186/1297-9686-46-23.

Daetwyler, H. D., R. Pong-Wong, B. Villanueva, and J. A. Woolliams. 2010. The impact of genetic architecture on genome-wide evaluation methods. Genetics 185:1021-1031. https://doi.org/10.1534/ genetics.110.116855.

Esfandyari, H., A. C. Sørensen, and P. Bijma. 2015a. A crossbred reference population can improve the response to genomic selection for crossbred performance. Genet. Sel. Evol. 47:76. https:// doi.org/10.1186/s12711-015-0155-z.

Esfandyari, H., A. C. Sørensen, and P. Bijma. 2015b. Maximizing crossbred performance through purebred genomic selection. Genet. Sel. Evol. 47:16. https://doi.org/10.1186/s12711-015-0099-3.

Fragomeni, B. O., D. A. L. Lourenco, S. Tsuruta, Y. Masuda, I. Aguilar, A. Legarra, T. J. Lawlor, and I. Misztal. 2015. Hot topic: Use of genomic recursions in single-step genomic BLUP with a large number of genotypes. J. Dairy Sci. 98:4090-4094. https://doi.org/ $10.3168 /$ jds.2014-9125.

Garcia, A. L. S., Y. Masuda, S. Tsuruta, S. Miller, I. Misztal, and D. Lourenco. 2020. Indirect predictions with a large number of genotyped animals using the algorithm for proven and young. J. Anim. Sci. 98:skaa154. https://doi.org/10.1093/jas/skaa154.

Gonzalez-Recio, O., M. P. Coffey, and J. E. Pryce. 2014. On the value of the phenotypes in the genomic era. J. Dairy Sci. 97:7905-7915. https://doi.org/10.3168/jds.2014-8125.

Guinan, F. L., D. Norman, and J. Dürr. 2019. Changes occurring in the breed composition of U.S. dairy herds. Interbull Bull. 55:11-16.

Habier, D., R. L. Fernando, and D. J. Garrick. 2013. Genomic BLUP decoded: A look into the black box of genomic prediction. Genetics 194:597-607. https://doi.org/10.1534/genetics.113.152207.

Harris, B. L., and D. L. Johnson. 2010. Genomic predictions for New Zealand dairy bulls and integration with national genetic evaluation. J. Dairy Sci. 93:1243-1252. https://doi.org/10.3168/jds.2009 -2619 .

Hidalgo, A. M., J. W. M. Bastiaansen, M. S. Lopes, B. Harlizius, M. A. M. Groenen, and D.-J. de Koning. 2015. Accuracy of predicted genomic breeding values in purebred and crossbred pigs. G3 (Bethesda) 5:1575-1583. https://doi.org/10.1534/g3.115.018119.

Illumina Inc. 2011a. GoldenGate Bovine3K Genotyping BeadChip. Accessed Sep. 29, 2020. http://support.illumina.com/content/dam/ illumina-marketing/documents/products/datasheets/datasheet _bovine3k.pdf.

Illumina Inc. 2011b. BovineSNP50 Genotyping BeadChip. Accessed Sep. 29, 2020. http://www.illumina.com/Documents/products/ datasheets/datasheet_bovine_snp50.pdf.

Legarra, A., I. Aguilar, and I. Misztal. 2009. A relationship matrix including full pedigree and genomic information. J. Dairy Sci. 92:4656-4663. https://doi.org/10.3168/jds.2009-2061.

Legarra, A., D. A. L. Lourenco, and Z. Vitezica. 2018. Bases for genomic predictions. Accessed Feb. 15, 2021. http://nce.ads.uga .edu/wiki/lib/exe/fetch.php?media=gsip.pdf.

Lopes, M. S., H. Bovenhuis, A. M. Hidalgo, J. A. M. van Arendonk, E. F. Knol, and J. W. M. Bastiaansen. 2017. Genomic selection for crossbred performance accounting for breed-specific effects. Genet. Sel. Evol. 49:51. https://doi.org/10.1186/s12711-017-0328-z. 
Lourenco, D. A., S. Tsuruta, B. Fragomeni, C. Chen, W. Herring, and I. Misztal. 2016. Crossbreed evaluations in single-step genomic best linear unbiased predictor using adjusted realized relationship matrices. J. Anim. Sci. 94:909-919. https://doi.org/10.2527/jas 2015-9748

Lourenco, D. A. L., A. Legarra, S. Tsuruta, D. Moser, S. Miller, and I. Misztal. 2018. Tuning indirect predictions based on SNP effects from single-step GBLUP. Interbull Bull. 53:48-53.

Misztal, I. 2016. Inexpensive computation of the inverse of the genomic relationship matrix in populations with small effective population size. Genetics. 202:401-409. https://doi.org/10.1534/genetics.115 .182089 .

Misztal, I., S. Tsuruta, D. Lourenco, I. Aguilar, A. Legarra, and Z. Vitezica. 2014. Manual for BLUPF90 Family of Programs. University of Georgia, Athens. http://nce.ads.uga.edu/wiki/lib/exe/fetch .php?media=blupf90_all2.pdf.

Olson, K. M., P. VanRaden, and M. Tooker. 2012. Multibreed genomic evaluations using purebred Holsteins, Jerseys, and Brown Swiss. J. Dairy Sci. 95:5378-5383. https://doi.org/10.3168/jds.2011-5006.

Pocrnic, I., D. A. Lourenco, C.-Y. Chen, W. O. Herring, and I. Misztal. 2019b. Crossbred evaluations using single-step genomic BLUP and algorithm for proven and young with different sources of data. J. Anim. Sci. 97:1513-1522. https://doi.org/10.1093/jas/skz042.

Pocrnic, I., D. A. Lourenco, Y. Masuda, A. Legarra, and I. Misztal. 2016a. The dimensionality of genomic information and its effect on genomic prediction. Genetics 203:573-581. https://doi.org/10 .1534/genetics.116.187013.

Pocrnic, I., D. A. L. Lourenco, Y. Masuda, and I. Misztal. 2016b. Dimensionality of genomic information and performance of the Algorithm for Proven and Young for different livestock species. Genet. Sel. Evol. 48:82. https://doi.org/10.1186/s12711-016-0261-6.

Pocrnic, I., D. A. L. Lourenco, Y. Masuda, and I. Misztal. 2019a. Accuracy of genomic BLUP when considering a genomic relationship matrix based on the number of the largest eigenvalues: A simulation study. Genet. Sel. Evol. 51:75. https://doi.org/10.1186/s12711 -019-0516-0.

Pryce, J. E., B. Gredler, S. Bolormaa, P. J. Bowman, C. Egger-Danner, C. Fuerst, R. Emmerling, J. Sölkner, M. E. Goddard, and B. J. Hayes. 2011. Short communication: Genomic selection using a multi-breed, across-country reference population. J. Dairy Sci. 94:2625-2630. https://doi.org/10.3168/jds.2010-3719.

Raymond, B., A. C. Bouwman, C. Schrooten, J. Houwing-Duistermaat, and R. F. Veerkamp. 2018. Utility of whole-genome sequence data for across-breed genomic prediction. Genet. Sel. Evol. 50:27. https: //doi.org/10.1186/s12711-018-0396-8.

Raymond, B., L. Yengo, R. Costilla, C. Schrooten, A. C. Bouwman, B. J. Hayes, R. F. Veerkamp, and P. M. Visscher. 2020. Using prior information from humans to prioritize genes and gene-associated variants for complex traits in livestock. PLoS Genet. 16:e1008780. https://doi.org/10.1371/journal.pgen.1008780.

Sargolzaei, M., J. P. Chesnais, and F. S. Schenkel. 2011. FImpute: An efficient imputation algorithm for dairy cattle populations. J. Dairy Sci. 94(E-Suppl. 1):421. (Abstr.)

Sevillano, C. A., J. Vandenplas, J. W. M. Bastiaansen, and M. P. L. Calus. 2016. Empirical determination of breed-of-origin of alleles in three-breed cross pigs. Genet. Sel. Evol. 48:55. https://doi.org/ 10.1186/s12711-016-0234-9.

Spelman, R. J., C. Ford, P. McElhinney, G. Gregory, and R. Snell. 2002. Characterization of the DGAT1 gene in the New Zealand dairy population. J. Dairy Sci. 85:3514-3517. https://doi.org/10 $.3168 /$ jds.S0022-0302(02)74440-8.
Steyn, Y., D. A. L. Lourenco, and I. Misztal. 2019. Genomic predictions in purebreds with a multibreed genomic relationship matrix. J. Anim. Sci. 97:4418-4427. https://doi.org/10.1093/jas/skz296.

Strandén, I., and E. A. Mäntysaari. 2013. Use of random regression model as an alternative for multibreed relationship matrix. J. Anim. Breed. Genet. 130:4-9. https://doi.org/10.1111/jbg.12014.

Thaller, G., W. Krämer, A. Winter, B. Kaupe, G. Erhardt, and R. Fries. 2003. Effects of DGAT1 variants on milk production traits in German cattle breeds. J. Anim. Sci. 81:1911-1918. https://doi .org/10.2527/2003.8181911x.

Toosi, A. R. L. Fernando, and J. C. M. Dekkers. 2010. Genomic selection in admixed and crossbred populations. J. Anim. Sci. 88:32-46. https://doi.org/10.2527/jas.2009-1975.

Vandenplas, J., M. P. L. Calus, C. A. Sevillano, J. J. Windig, and J. W. M. Bastiaansen. 2016. Assigning breed origin to alleles in crossbred animals. Genet. Sel. Evol. 48:61. https://doi.org/10.1186/ s12711-016-0240-y.

VanRaden, P. M. 2008. Efficient methods to compute genomic predictions. J. Dairy Sci. 91:4414-4423. https://doi.org/10.3168/jds .2007-0980.

VanRaden, P. M., M. E. Tooker, T. C. S. Chud, H. D. Norman, J. H. Megonigal Jr., I. W. Haagen, and G. R. Wiggans. 2020. Genomic predictions for crossbred dairy cattle. J. Dairy Sci. 103:1620-1631. https://doi.org/10.3168/jds.2019-16634.

Vukasinovic, N., N. Bacciu, C. A. Przybyla, P. Boddhireddy, and S. K. DeNise. 2017. Development of genetic and genomic evaluation for wellness traits in US Holstein cows. J. Dairy Sci. 100:428-438. https://doi.org/10.3168/jds.2016-11520.

Wang, H., I. Misztal, I. Aguilar, A. Legarra, and W. M. Muir. 2012. Genome-wide association mapping including phenotypes from relatives without genotypes. Genet. Res. (Camb.) 94:73-83. https:// doi.org/10.1017/S0016672312000274.

Wei, M., and J. H. J. van der Werf. 1994. Maximizing genetic response in crossbreds using both purebred and crossbred information. Anim. Sci. 59:401-413. https://doi.org/10.1017/S0003356100007923.

Wellmann, R. 2019. Optimum contribution selection for animal breeding and conservation: The R package optiSel. BMC Bioinformatics 20:25. https://doi.org/10.1186/s12859-018-2450-5.

Wellmann, R. 2020. Package 'optiSel' manual. https://cran.r-project .org/web/packages/optiSel/optiSel.pdf.

Wiggans, G. R. 1997: Genetic evaluation systems in the United States. Accessed Oct. 26, 2020. https://aipl.arsusda.gov/publish/other/ 1997/conf_isap97_19.html.

Wiggans, G. R., P. M. VanRaden, E. L. Nicolazzi, M. E. Tooker, J. H. Megonigal Jr., and L. M. Walton. 2019. Extending genomic evaluation to crossbred dairy cattle - US implementation. Interbull Bull. 55:46-49.

\section{ORCIDS}

Y. Steyn ๑ https://orcid.org/0000-0001-5467-9555

D. Gonzalez-Pena (i) https://orcid.org/0000-0003-4846-7569

Y. L. Bernal Rubio @ https://orcid.org/0000-0002-6583-7712

N. Vukasinovic @ https://orcid.org/0000-0002-5514-826X

D. A. L. Lourenco @ https://orcid.org/0000-0003-3140-1002

I. Misztal ๑ https://orcid.org/0000-0002-0382-1897 